\title{
CORNEAL ENDOTHELIAL EVALUATION AFTER COLLAGEN CORNEA CROSS-LINKING
}

\author{
By

\begin{abstract}
Ahmed Kamal Abd El-Fatah Fouda, Mohamed Khedr Mohamed and Omar Hassan Salama
\end{abstract}

Department of Ophthalmology, Faculty of Medicine, Al-Azhar University, Cairo, Egypt

Corresponding Author: Ahmed Kamal Abd El-Fatah Fouda,

E-mail: ahmed.kamal.fouda@gmail.com

\begin{abstract}
Background: Keratoconus is a progressive disorder in which the cornea assumes a conical shape secondary to stromal thinning and protrusion. Both eyes are affected, at least on topographical imaging, in almost all cases. Collagen cross-linking (CXL) is a bioengineering technique to increase the mechanical stability of the cornea in corneal ectatic disorders such as keratoconus (KC). Endothelial changes were reported after CXL.
\end{abstract}

Objective: To evaluate the corneal endothelial changes following cross linking for treatment of keratoconus using corneal specular microscopy and corneal volume measurements.

Patient and methods: A prospective, non-randomized interventional case series was conducted on 20 eyes diagnosed with progressive keratoconus. Patients were evaluated for endothelial changes using corneal specular microscopy and for Keratometric and corneal volume measurements using Pentacam. Measurements were done preoperatively and 6 months postoperatively. This study was carried out during the period from January 2020 till July 2020 at al-Hussein university hospital.

Results: The mean age of the studied patients was $20.50 \pm 0.51$ years. Of total 10 patients, there were 3 males $(30 \%)$ and 7 females $(70 \%)$. There was significant reduction in K1, K2, K max, thinnest location pachymetry, corneal volume, endothelial cell density (CD) and hexagonal cells percentage (Hex\%) postoperatively, while Coefficient of variation (CV\%) changes were insignificant. Results showed that CD change and Hex\% change had reverse proportional correlation with corneal volume change, while, central corneal thickness change has positive proportional correlation with corneal volume change.

Conclusion: CXL significantly flattened keratoconic corneas with significant reported endothelial changes. The study could not confirm the value of corneal volume change as a potential indicator for widespread endothelial dysfunction after CXL.

Keywords: Collagen Cornea Cross-Linking, Corneal volume, Keratoconus, Specular microscopy.

\section{INTRODUCTION}

Keratoconus is a progressive disorder in which the cornea assumes a conical shape secondary to stromal thinning and protrusion. Both eyes are affected at least on topographical imaging, in almost all cases. The hallmark of keratoconus is central or paracentral stromal thinning accompanied by apical protrusion and irregular astigmatism. The disease tends to progress during the adolescent years and into the mid-20s and 30s, although progression can occur at any time (Martínez-Abad and Pinero, 2017).

According to Global Consensus on Keratoconus and Ectatic Diseases (2015), there is no consistent or clear definition of ectasia progression (Gomes et al., 2015). 
Progression was defined by a consistent change in at least two of these parameters: anterior corneal surface steepening, posterior corneal surface steepening and/or thinning or changes in the pachymetric rate of change. According to the panel, agreement on specific quantitative data to define progression is lacking (Duncan et al., 2016).

For more than a decade, corneal crosslinking (CXL) has been considered as the only method for improving corneal biomechanical power. Corneal collagen cross-linking (CXL) is a less-invasive treatment that aimed to strengthen biomechanical stability in eyes with keratectasia (Rubinfeld et al., 2019). The technique of corneal collagen crosslinking consists of photopolymerization of stromal fibers by the combined action of a photosensitizing substance (riboflavin or vitamin B2) and ultraviolet A rays (UVA) from a solid-state UVA source (El-Massry et al., 2017). Photopolymerization increases the rigidity of corneal collagen and its resistance to keratectomies. The cross-linking effect is not distributed homogenously over the corneal depth. The stiffening effect is concentrated in the anterior 200 to 300 microns of the cornea due to the high absorption of UV light in this area (Gatinel, 2017). The effect of CXL depends on the total energy doses, so the same therapeutic effect can be theoretically obtained by increasing the intensity and decreasing the time of irradiation. For this reason, secondgeneration CXL devices have been developed to increase the intensity and optimized beam shaping to accelerate the treatment (Kang et al., 2020).
Complications of CXL included corneal haze $(90 \%)$, sterile infiltrates (7$10 \%$ ) and corneal edema (especially in thin corneas). There can be continued progression of KC. Infective keratitis, reactivation of herpes simplex virus infection and endothelial cell loss are rarely seen. Nevertheless, the current stand in clinical practice remains that CXL is a safe and efficacious treatment modality for corneal ectatic diseases and holds promise for arresting their progression (Gupta, 2017). Effects of CXL on endothelial layer have been reported by many authors (Helal et al., 2018, El-Sayed et al., 2019 and Rubinfeld et al., 2019).

This study aimed to evaluate the corneal endothelium following cross linking for treatment of keratoconus both anatomically by corneal specular microscopy and functionally by corneal volume measurements.

\section{PATIENTS AND METHODS}

A prospective, non-randomized interventional case series was conducted on 20 eyes diagnosed with progressive keratoconus. This study was carried out during the period from January 2020 till July 2020 at al-Hussein University Hospital

Ethical consideration: All participants signed written informed consents that explain the aim of study before the study initiation. All patients were instructed about the technique and possible complications of the procedure. Approval was obtained from the ethical committee in Faculty of Medicine, Al-Azhar University, Cairo, Egypt. 
Inclusion criteria: Patients aged from 18 to 40 years, with progressive keratoconus based on Global Consensus on Keratoconus and Ectatic Diseases (2015) criteria. Both genders were included.

Exclusion criteria: Patients with history of contact lens wear, herpetic keratitis or ocular refractive surgery, severe allergic conjunctivitis, severe dry eye, corneal opacity, glaucoma, pseudoexfoliation, cataract, or vitreoretinal disorders were excluded. Corneas thinner than $400 \mu$ and patients with autoimmune diseases or diabetes were also excluded.

All patients subjected to detailed history taking and thorough ophthalmic examination. History included: age, medical history, surgical history, previous trauma and last time they used contact lens if they were contact lens wearer. Ophthalmic examination was done before Pentacam including: Uncorrected visual acuity (UCVA) using Snellen chart, Best Corrected Visual Acuity (BCVA) for distance, anterior segment examination by slit lamp biomicroscopy, fundus examination and intraocular pressure, Investigations: General medical investigations for systemic evaluation were performed including blood sugar, complete blood count, liver function tests, kidney function tests and ECG. Scheimpflug imaging: we used Oculus Pentacam $^{\circledR}$ HR, examination was performed in dim illumination, patients were positioned comfortably in front of the instrument and were asked to blink frequently before examination. The following parameters were recorded: $\mathrm{K} 1$, K2, Kmax, thinnest location pachymetry and corneal volume. Specular microscopy was done by Topcon ${ }^{\circledR}$ SP-1P.
Using panorama mode, 3 images from the central cornea were captured then the machine combines the 3 images giving a wide analysis about the central corneal endothelium. The following data were recorded: mean cell density (CD) cell/mm2, coefficient of variation (CV \%) in cell size and percentage of hexagonal cells (HEX\%). Pentacam imaging and specular microscopy were performed preoperatively and 3 months postoperatively.

Surgical procedure: All patients were treated with UVA/riboflavin CXL under sterile conditions in the operating theater. First, periocular skin was scrubbed with $10 \%$ povidone-iodine (Betadine, Mondy pharma) and a sterile drape was applied. Topical anesthetic drops were used (benoxinate hydrochloride $0.4 \%$ ) twice: one before starting the procedure and once before UVA exposure. A micro sponge soaked with ethyl alcohol $20 \%$ was applied onto central part of the cornea for 15 seconds. Then by using hockey stick spatula, an $8.0 \mathrm{~mm}$ diameter of central corneal epithelium was removed. The isoosmolar $0.1 \%$ riboflavin solution (Mediocross M, Germany) was instilled every $5 \mathrm{~min}$ for $30 \mathrm{~min}$. Then UVA irradiation was applied for 30 minutes using UVA system devised (OPTO X Link TM). Riboflavin solution was applied every 5 min during the UVA irradiation time. At the end of the procedure, soft bandage contact lens was placed for 5 days till complete re-epithelization. Postoperative topical treatment included antibiotics (Gatifloxacin 0.3\%), steroid (Prednisolone acetate $1 \%$ ) eye drops q.i.d for 4 weeks with frequent topical lubricant eye drops (Carboxymethylcellulose Sodium 0.5\%) for 12 weeks. 
Statistical design: Results were tabulated and statistically analyzed using standard computer program using Microsoft Excel 2017 and SPSS V.25 program for Microsoft Windows 10. Description of data was in the form of mean $( \pm)$ SD for quantitively data, and frequency and proportion for qualitative data. Paired ttest, and Spearman correlation coefficient test were used. $\mathrm{P}<0.05$ was considered statistically significant.

\section{RESULTS}

The mean age of the studied patients was $20.50 \pm 0.51$ years. Of total 10 patients, there were 3 males (30\%) and 7 females (70\%) (Table 1).

Table (1): Distribution of the studied patients regarding demographic data

SD: standard deviations

\begin{tabular}{|l|c|c|}
\hline Items & \multicolumn{2}{|c|}{ The studied patients (n=20 eye) } \\
\hline Age & & $20.50 \pm 0.51$ \\
-Mean \pm SD & & $20.00-21.00$ \\
-Range & No. & \% \\
\hline & & $30.0 \%$ \\
\hline Gender & 3 & $70.0 \%$ \\
-Male & 7 & $50.0 \%$ \\
-Female & & $50 \%$ \\
\hline Eye Side & 10 & \\
-Right & 10 & \\
-Left & & \\
\hline
\end{tabular}

There was a significant reduction in keratometric measurements: mean preoperative $\mathrm{K} 1$ was $43.41 \pm 1.28 \mathrm{D}$, Vs. $42.11 \pm 1.59 \mathrm{D}$ postoperatively $(\mathrm{P}=0.001)$, mean preoperative $\mathrm{K} 2$ was $49.20 \pm 2.83 \mathrm{D}$, Vs. $47.28 \pm 3.16 \mathrm{D}$ postoperatively $(\mathrm{P}=0.001)$ and mean preoperative Kmax was $52.55 \pm 5.39 \mathrm{D}$, Vs. $51.5=20 \pm 3.12 \mathrm{D}$ postoperatively $\quad(\mathrm{P}=0.036)$. Thinnest location pachymetry reported significant reduction from $474.50 \pm 6.99 \mu$ preoperatively to $471.80 \pm 4.51 \quad \mu$ postoperatively $\quad(\mathrm{P}=0.002)$. Corneal volume also reported significant reduction from $56.64 \pm 1.55 \mathrm{~mm} 3$ preoperatively to
$56.06 \pm 1.76 \quad \mathrm{~mm} 3$ postoperatively $(\mathrm{P}=0.005)$.

Regarding specular microscopy: mean cell density (CD) reported significant reduction from $3066.20 \pm 167.23 \mathrm{cell} / \mathrm{mm} 2$ preoperatively to $2413.70 \pm 1230.22$ cell $/ \mathrm{mm} 2$ postoperatively with $(\mathrm{P}=0.048)$, similarly, mean hexagonal cells percentage (HEX \%) reported significant reduction from $64.31 \pm 5.11$ preoperatively to $52.80 \pm 3.61$ postoperatively $(\mathrm{P}=0.016)$. Mean coefficient of variation $(\mathrm{CV})$ showed no significant change $(\mathrm{P}=0.76)$ (Table 2). 
Table (2): Comparison between patients pre and postoperative regarding keratometric values, thinnest location, corneal volume values and microscopic values

\begin{tabular}{|c|c|c|c|c|c|}
\hline \multirow{2}{*}{$\begin{array}{r}\begin{array}{r}\text { Collagen } \\
\text { Cornea } \\
\text { Cross- } \\
\text { Linking }\end{array} \\
\text { Parameters }\end{array}$} & \multicolumn{2}{|c|}{$\mathbf{N}=\mathbf{2 0}$} & \multirow[b]{2}{*}{$\begin{array}{c}P \\
\text { value }\end{array}$} & \multicolumn{2}{|c|}{$95 \% \mathrm{CI}$} \\
\hline & Before & After & & Lower & Upper \\
\hline \multicolumn{6}{|c|}{ Keratometric values } \\
\hline $\begin{array}{l}\text { K1 } \\
\text {-Mean } \pm \text { SD }\end{array}$ & $43.41 \pm 1.28$ & $42.11 \pm 1.59$ & 0.001 & 0.229 & 0.730 \\
\hline $\begin{array}{l}\text { K2 } \\
- \text { Mean } \pm \text { SD }\end{array}$ & $49.20 \pm 2.83$ & $47.28 \pm 3.16$ & 0.001 & -0.234 & 0.846 \\
\hline $\begin{array}{l}\text { K max } \\
- \text { Mean } \pm \text { SD }\end{array}$ & $52.55 \pm 5.39$ & $51.20 \pm 3.12$ & 0.036 & -0.022 & 0.422 \\
\hline $\begin{array}{l}\text { Thinnest } \\
\text { local } \\
\text {-Mean } \pm \text { SD }\end{array}$ & $474.50 \pm 6.99$ & $471.80 \pm 4.51$ & 0.002 & 1.150 & 4.249 \\
\hline $\begin{array}{l}\text { Corneal } \\
\text { Volume } \\
\text {-Mean } \pm \text { SD }\end{array}$ & $56.64 \pm 1.55$ & $56.06 \pm 1.76$ & 0.005 & 0.194 & 0.965 \\
\hline \multicolumn{6}{|c|}{ Specular microscopic } \\
\hline $\begin{array}{l}\text { CD } \\
- \text { Mean } \pm \text { SD }\end{array}$ & $3066.20 \pm 167.23$ & $2413.70 \pm 1230.22$ & 0.048 & 6.819 & 1298.18 \\
\hline $\begin{array}{l}\text { CV } \\
- \text { Mean } \pm \text { SD }\end{array}$ & $29.40 \pm 4.24$ & $30.12 \pm 1.05$ & 0.76 & -2.540 & 2.54 \\
\hline $\begin{array}{l}\text { Hex } \\
\text {-Mean } \pm \text { SD }\end{array}$ & $64.31 \pm 5.11$ & $52.80 \pm 3.61$ & 0.016 & -83.10 & -0.964 \\
\hline
\end{tabular}

SD: standard deviations, $\mathrm{K}$ max = maximum keratometric value, CD: Cell density, CV: Coefficient of variation, HEX: Hexagonal cells percentage.

Changes in specular microscopic were tested for correlation to changes in corneal volume. Results showed that $\mathrm{CD}$ and $\mathrm{HEX} \%$ changes had significant negative correlation with corneal volume change
(P-value: 0.04 and 0.001 respectively), while thinnest location pachymetry change had significant positive correlation with corneal volume change $(\mathrm{P}=0.027)$ (Table 3, Figures 1, $2 \& 3$ ).

Table (3): Correlation between corneal volume change and specular microscopic change

\begin{tabular}{|l|c|c|}
\hline Changes Corneal volume change & $\mathrm{r}^{\mathrm{s}}$ & P value \\
\hline Cell density change & -0.462 & 0.040 \\
\hline Coefficient of variation change & -0.140 & 0.955 \\
\hline Hexagonal cells change & -0.611 & 0.001 \\
\hline
\end{tabular}

$r^{s}$ : Correlation coefficient 


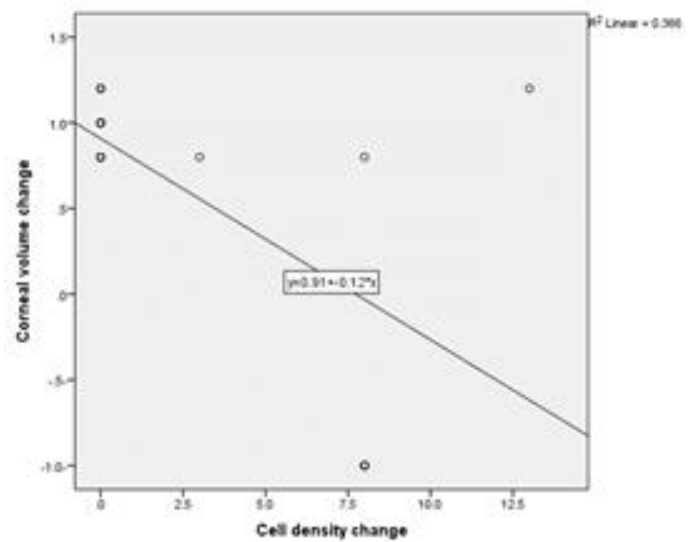

Fig. (1): Correlation between comeal volume change and cell density change.

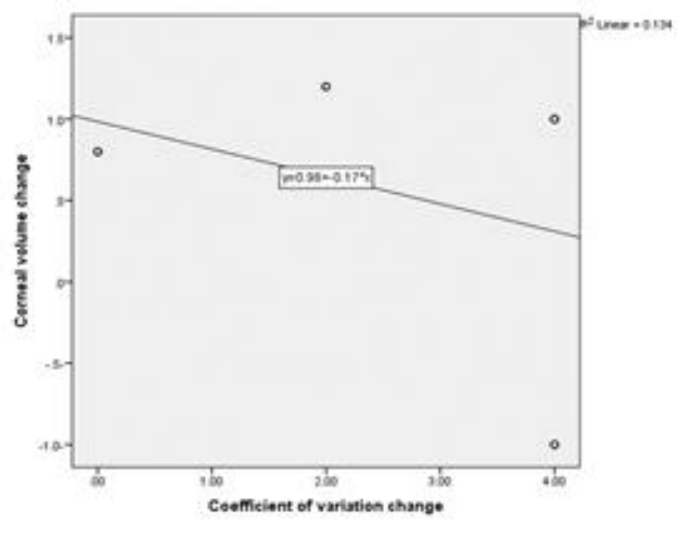

Fig. (2): Correlation between comeal volume change and coefficient of variation change.

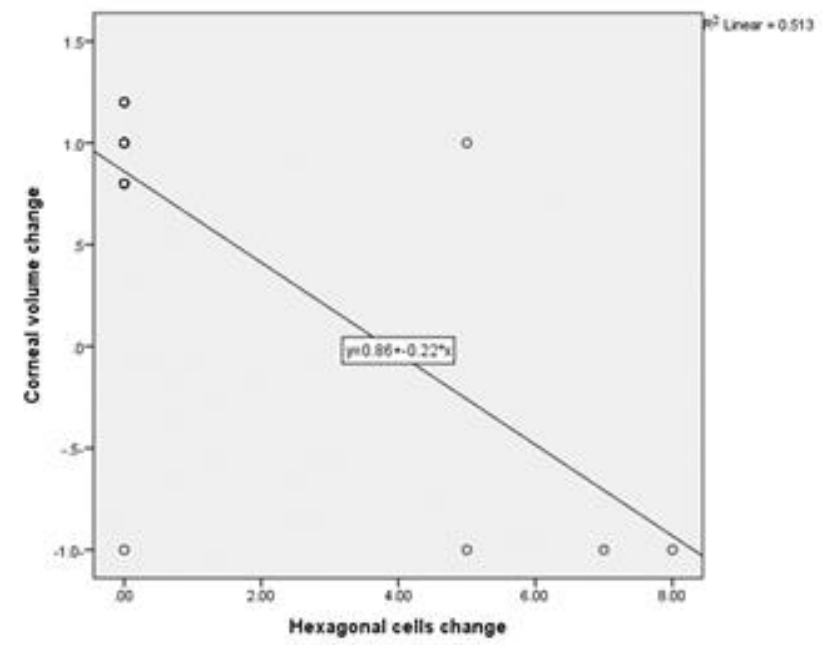

Fig. (3): Correlation between comeal volume change and hexagonal change.

\section{DISCUSSION}

Despite its general safety, many significant risks have been reported with this standard CXL protocol including infections, sterile keratitis, edema, scarring, and rarely corneal perforations. These complications may lead to further loss of vision or even loss of the eye. Moreover, associated pain and reduced vision for several weeks after standard CXL delay postoperative patients' recovery. These complications are mainly due to surgical removal of corneal epithelial protective layer and usually persist until this layer heals (Dhawan et al., 2011). Thus, less invasive alternatives were tried to replace standard CXL protocol to avoid these complications. Transepithelial or Epi-on CXL using various permeation enhancers, iontophosis or nanotechnology were described to allow stromal hydration with riboflavin through an intact epithelium (Taneri et al., 2014). Partial surgical debridement and epithelial disruption techniques were also described as alternatives for standard CXL (Razmjoo et al., 2014). In this study, we used the standard CXL protocol as other 
techniques showed less favorable results and none was completely safe.

Traditionally, assessment of corneal endothelium based on specular microscopy. Study of cell density and morphology provides important information on corneal endothelial function and vitality and largely become an accepted method in practice and research to provide valuable information on this layer (Benetz et al., 2011). Endothelial mosaic alterations are signs of surgical trauma and are essential in evaluating the safety of various surgical techniques (Moshirfar et al., 2014).

Modern imaging techniques allowed detailed study of the various anterior segment parameters. Many authors proposed the corneal volume as a functional parameter for endothelial evaluation. Based on the fact that the main function of corneal endothelium is to keep dehydration of corneal stroma; once compromised corneal volume increases. Furthermore, corneal volume is measured over a larger area in comparison to specular microscopy that allows evaluating endothelial function over a wide area.

Suzuki et al. (2010) introduced a corneal volume Pentacam ${ }^{\circledR}$ assay as a potentially new method for assessing phacoemulsification induced corneal endothelial damage. Later on, Suzuki et al. (2011) reported that the increased corneal volume noticed early within the first week in the 3-mm diameter area, almost returned to the preoperative values at 1 month follow up. Conversely, 10-mm corneal volumes at one month were still considerably higher compared with the preoperative values.
Many authors reported short term changes in central corneal thickness (Doganay et al., 2010 and Fares et al., 2012) and corneal volume (Benetz et al., 2011) associated with endothelial cell loss due to phacoemulsification induced surgical trauma. Most corneas returned to normal thickness by 1 month postoperatively. In Egyptian patients, Elwerdany, et al. (2020) studied corneal volume changes after phacoemulsification. They reported mean preoperative corneal volume of 56.70 $\mathrm{mm} 3$ that was significantly increased to $59.27 \mathrm{~mm} 31$ month postoperatively.

To our knowledge, this is the first report investigating the corneal volume as a potential functional indicator of corneal endothelium after CXL. Unlike phacoemulsification, CXL includes further direct surgical impact on corneal stroma; so, a longer postoperative follow up period should be permitted for corneal stromal edema to resolve. Long lasting corneal volume changes at 3 months postoperatively over a wide diameter (10 $\mathrm{mm}$ ) area may be an indicator for a widespread endothelial dysfunction following CXL.

We have excluded patients with factors known to affect the corneal endothelial cell density and morphology either systemic like: Type 2 Diabetes Mellitus (Rachapalle et al., 2012) or local like prolonged contact lens wear and phakic intraocular lens implantation (Moshirfar et al., 2014), glaucoma and pseudoexfoliation (Kwon et al., 2016).

We have also excluded patients with thin corneas to ensure safety of deeper structures, i.e. endothelium, lens, or retina. The limit of 400 um CCT was generally 
considered safe for CXL (Wollensak et al., 2010). Kymionis et al. (2012) documented a significant decrease in the endothelial $\mathrm{CD}$ after performing standard CXL in thin corneas despite positive clinical outcomes. Thin, de-epithelialized corneas, soaked with a hypo-osmolar solution can get double CCT pachymetry. CXL for such cases showed stabilization of keratectasia and no detectable stromal scarring but with decreased endothelial CD. Hypoosmolar riboflavin solutions proved not a safe, optimal solution for corneas $<330$ mm (Hafezi, 2011).

As such protocols were not agreed between authors as regard safety and efficacy; we adhered to standard CXL protocol and the limitation of $400 \mu$ thinnest location pachymetry.

Reports about corneal thickness changes after CXL showed variable results. Chow et al. (2015), reported decreased mean central corneal thickness at 1 year follow up after both conventional and accelerated CXL. In the conventional CXL group, thinnest corneal location and central corneal thickness decreased significantly post operatively from 447.89 and $464.12 \mu$ to 417.97 and $440.56 \mu$, respectively. In the accelerated CXL group, thinnest corneal location and central corneal thickness decreased significantly post operatively from 466.39 and $482.74 \mu$ to 444.02 and $460.10 \mu$, respectively.

An earlier report by Rosa et al. (2010) documented a statistically significant decrease in central pachymetry 1month after treatment that tended to increase during the 24 months follow-up, but, without reaching the preoperative values.
On the contrary, Vinciguerra et al. (2010) reported pupil center pachymetry to decrease in first 6 months, and then approached preoperative values after 12 months. After 24 months values were higher than preoperative values, but changes were statistically insignificant compared to preoperative data. Kymionis et al. (2012) reported that after more than 5 years follow up period, the mean central corneal thickness (CCT) did not change significantly at any postoperative interval.

In Egyptian patients, Tawakol et al. (2019) reported about $11.5 \mu$ decrease of CCT, 4 months after standard CXL. Omar and Zein (2019) demonstrated that, corneal thickness showed significant changes 1 year after accelerated CXL. After the same CXL protocol, Mansour et al. (2020) reported about $9 \mu$ decrease of mean CCT, only 6 weeks postoperatively.

In our study, using Pentacam, mean thinnest location pachymetry preoperatively decreased about $2.7 \mu$ (from 474.50 to $471.80 \mu$ ) 3 months postoperatively, a result that was statistically significant .

Many authors documented specular microscopic changes after CXL. While, only few reports documented corneal volume changes.

Vinciguerra et al. (2012) reported early drop in CD within few months postoperatively that then increased gradually afterwards. The difference between baseline and 24 months was not statistically significant, indicating that CXL did not induce endothelial cell damage in the 2-year follow-up period. Kymionis et al. (2012) reported that the mean CD did not change significantly at any postoperative interval nor at the last 
follow-up visit 5 years after CXL. Similarly, Rechichi et al. (2013) showed that there was a statistically insignificant difference in $\mathrm{CD}$ after 1 year.

On the other hand, Razmjoo et al. (2014) found that there was a significant reduction in $\mathrm{CD}$, but this reduction was low. In addition, the cell size (polymegathism) represented by the CV\% was significantly increased. Also, they found the preoperative hexagonal cells percentage representing pleomorphism, was $54.14 \%$, whereas the postoperative percentage was $54.55 \%$, indicating no significant change in cell shape.

On comparing standard and accelerated CXL, Chow et al. (2015) reported that there was a significant decrease in endothelial cell density after accelerated CXL but not after conventional CXL. However, the mean loss of cells was clinically inconsequential and comparable between both groups.

In Egyptian patients, 1 month after standard CXL, Helal et al. (2018) documented insignificant decrease of $\mathrm{CD}$ and insignificant increase in $\mathrm{CV} \%$ and hexagonal cells percentage, while Elgazzar et al. (2020) reported decrease of $\mathrm{CD}, \mathrm{CV} \%$, and hexagonal cells percentage, yet, all changes were statistically insignificant.

The current study showed significant reduction in cell density $(C D)$ values from 3066.20 cells $/ \mathrm{mm} 2$ preoperatively, to 2413.70 cells $/ \mathrm{mm} 2$ postoperatively, and significant reduction of the mean Hex\% from $64.31 \%$ preoperatively to $52.8 \%$ postoperatively. Concerning $\mathrm{CV} \%$, the mean values were $29.40 \%$ preoperatively, and $29.40 \%$ postoperatively.
Regarding changes in corneal volume, Vinciguerra et al. (2010) reported that total corneal volume decreased from 59.37 $\mathrm{mm} 3$ to $57.17 \mathrm{~mm} 3$ at 12 months, then slightly increased to $58.28 \mathrm{~mm} 324$ months after treatment, compared with the 12-month values. Associated pupil center pachymetry measurements at baseline, 12 months and 24 months were $490.68 \mu$, $470.09 \mu$ and $479.91 \mu$ respectively.

Omar and Zien (2019) reported significant decrease in corneal volume from $57.97 \mathrm{~mm} 3$ to $56.91 \mathrm{~mm} 3$ one year after epithelium off accelerated CXL. In this study, decreased corneal volume was associated with decreased central corneal thickness by mean of about $10 \mu$ and thinnest location also decreased significantly by mean difference of $10.38 \mu$.

In our study, mean corneal volume was $56.64 \mathrm{~mm} 3$ preoperatively that decreased significantly to $56.06 \quad \mathrm{~mm} 3$ postoperatively. On correlating changes in mean corneal volume to changes in specular microscopic parameters, there was a significant positive correlation to changes in thinnest corneal pachymetry and a significant negative correlation to changes in $\mathrm{CD}$ and $\mathrm{Hex} \%$, while correlation to $\mathrm{CV} \%$ changes was insignificant. The positive correlation to CCT was expected and understood.

The main hypothesis of this study was that if standard CXL significantly affects endothelial functions, it would be associated with increased corneal volume. The negative correlation of volume changes (as an indicator for widespread endothelial dysfunction) to $\mathrm{CD}$ and $\mathrm{Hex} \%$ changes (as an endothelial anatomical 
evaluation factors) may support this hypothesis.

The decreased mean value of corneal volume at the end of the study, in comparison to preoperative value, may be against our hypothesis. However, this can be explained by associated decreased thinnest corneal pachymetry and the relatively short follow up period. In aforementioned report, Rosa et al. (2010) documented a statistically significant decrease in central corneal thickness 1 month after treatment that tended to increase during the 24 months follow-up; they explained their results by stromal keratocyte repopulation or artifacts in CCT measurements due to postoperative corneal edema. The corneal volume was not documented in that report, but increased central corneal thickness is supposed to be associated with increased corneal volume.

Similarly, Vinciguerra et al. (2010) documented increased pupil center pachymetry and corneal volume greater than preoperative values by 24 months postoperatively. The reported increase in corneal volume after long follow up period may be explained by long term endothelial dysfunction with associated corneal hydration.

Three months follow up period was too short to confirm whether corneal volume changes can be reliably considered an indicator for endothelial dysfunction.

\section{CONCLUSION}

Corneal cross linking significantly affects corneal endothelium. Specular microscopy remains the gold standard for corneal endothelial evaluation after CXL. Longer follow up periods and larger sample size are required for better evaluation of corneal volume as an indicator for endothelial dysfunction after CXL.

\section{REFERENCES}

1. Benetz BA, Yee R, Bidros M and Lass J. (2011): Specular microscopy. In: Cornea; Fundamentals, diagnosis and management, Volume 1: Fundamentals and Medical Aspects of Cornea and External Disease, Part II: Examining and Imaging the Cornea and External Eye, Section 3: Imaging Techniques of the Cornea, Chapter 14. Third edition, Pbl. Elsevier Health Sciences, Mosby, Cincinnati OH, Pp. 427-477.

2. Chow VW, Chan TC, Yu M, Wong VW and Jhanji V. (2015): One-year outcomes of conventional and accelerated collagen crosslinking in progressive keratoconus. Scientific Reports, 5:14425-32.

3. Dhawan S, Rao $K$ and Natrajan $S$. (2011): Complications of corneal collagen crosslinking. J Ophthalmol., 11: 9015-19.

4. Doganay S, Firat PB, Emre S and Yologlu S. (2010): Evaluation of anterior segment parameter changes using the Pentacam after uneventful phacoemulsification. Acta Ophalmologica., 88(5): 601-606.

5. Duncan JK, Belin MW and Borgstrom M. (2016): Assessing progression of keratoconus: novel tomographic determinants. Eye and Vis., 3: 6-12.

6. Elgazzar AF, Eid RS and Aly MM. (2020): Corneal endothelial changes evaluation using specular microscope following collagen cross-linking in treatment of keratoconus. Delta Journal of Ophthalmology, 21(3):153-59. 
7. El-Massry AA, Dowidar AM, Massoud TH and Tadros BG. (2017): Evaluation of the effect of corneal collagen crosslinking for keratoconus on the ocular higher-order aberrations. Clinical Ophthalmology, 11:1461-66.

8. El-Sayed A, Abdallah AM, Ammar HG and Sayed KM. (2019): Changes in Endothelial Specular Microscopy findings before and after Corneal Crosslinking. Sohag Medical Journal, 23(3):83-7.

9. Elwerdany MA, Shelil AE and Salama OH. (2020): Evaluation of Corneal Endothelium Function by Combined Measurement of Corneal Volume and Specular Microscopy after Phacoemulsification. AIMJ, 1(4): 25-30.

10. Fares U, Otri AM, Al-Aqaba MA and Dua HS. (2012): Correlation of central and peripheral corneal thickness in healthy corneas. Cont Lens Anterior Eye, 35:39-45.

11. Gatinel D. (2017): Reevaluating the Effect-iveness of Corneal Collagen Cross-linking and Its True Biomechanical Effect in Human Eyes. Int $\mathbf{J}$ Kerat Ect Cor Dis., 6(1):3 4 - 41.

12. Gomes JA, Tan D, Rapuano CJ, Belin MW, Ambrósio Jr $R$ and Guell JL. (2015): Global consensus on keratoconus and ectatic disease. Cornea, 34:359-69.

13. Gupta Y. (2017): Corneal Collagen Crosslinking for Keratoconus: An update. The Official Scientific Journal of Delhi Ophthalmological Society, 28(1):62-4.

14. Hafezi F. (2011): Limitation of collagen cross-linking with hypoosmolar riboflavin solution: failure in an extremely thin cornea. Cornea, 30:917919

15. Helal NE, Elewa LS, Mohamed RS and Hamdy KM. (2018): Evaluation of
Corneal Endothelial Changes Using Specular Microscope before and after Collagen Cross Linking for the Treatment of Keratoconus. The Medical Journal of Cairo University, 86:49-53.

16. Kang Y, Li S, Liu C, Xu M, Shi S and Liu Y. (2020): Accelerated Epitheliumoff Corneal Cross-linking with High Ultraviolet Energy Dose $(7.2 \mathrm{~J} / \mathrm{cm} 2)$ for Progressive Keratoconus: 2-Year Results in a Chinese Population. Journal of Refractive Surgery, 36(11):731-9.

17. Kwon JW, Cho KJ, Kim HK, Lee JK, Gore PK, McCartney MD and Chuck RS. (2016): Analyses of Factors Affecting Endothelial Cell Density in an Eye Bank Corneal Donor Database. Cornea, 35(9): 1206-1210.

18. Kymionis GD, Portaliou DM and Diakonis VF. (2012): Corneal collagen cross-linking with riboflavin and ultraviolet-A irradiation in patients with thin corneas. Am J Ophthalmol., 153:2428.

19. Mansour AH, Mohamed HE and Khaled A. (2020): Evaluation of corneal endothelial cell changes following modified corneal collagen accelerated pulsed cross-linking in moderate keratoconus. Journal of Ophthalmology and Related Sciences, 4(1):30-6.

20. Martínez-Abad A and Pinero DP. (2017): New perspectives on the detection and progression of keratoconus. Journal of Cataract \& Refractive Surgery, 43(9):1213-27.

21. Moshirfar M, Imbornoni LM, Ostler EM and Muthappan V. (2014): Incidence rate and occurrence of visually significant cataract formation and corneal decompensation after implantation of Verisyse/Artisan phakic intraocular lens. Clin Ophthalmol., 8: 711-16. 
22. Omar IA and Zein HA. (2019): Accelerated Epithelium-Off Corneal Collagen Cross-Linking for Keratoconus: 12-Month Results. Clinical Ophthalmology, 2019; 13:2385.

23. Rachapalle RS, Rajiv $R$ and Tarun $S$. (2012): Changes in the Corneal Endothelial Cell Density and Morphology in Patients with Type 2 Diabetes Mellitus. Cornea, 31(10):111922.

24. Razmjoo H, Rahimi B, Kharraji M and Peyman A. (2014): Corneal haze and visual outcome after collagen crosslinking for keratoconus: a comparison between total epithelium off and partial epithelial removal methods. Adv Biomed Res., 3:221-26.

25. Rechichi M, Daya S, Scorcia V, Meduri A and Scorcia G. (2013): Epithelial-disruption collagen crosslinking for keratoconus: one-year results. Journal of Cataract \& Refractive Surgery, 39(8):1171-8.

26. Rosa N, Lanza M, Borrelli M, Polito B, Filosa ML and De Bernardo $M$. (2010): Comparison of central corneal thickness measured with Orbscan and Pentacam. Journal of Refractive Surgery, 23(9):895-9.

27. Rubinfeld RS, Caruso $C$ and Ostacolo C. (2019): Corneal cross-linking: the science beyond the myths and misconceptions. Cornea, 38(6):780-90.

28. Suzuki H, Oki K, Takahashi K, Shiwa T and Takahashi H. (2010): Functional evaluation of corneal endothelium by combined measurement of corneal volume alteration and cell density after phacoemulsification. J Cataract Refract Surg., 33: 2077-2082.

29. Suzuki H, Takahashi H, Hori J, Hiraoka M, Igarashi T and Shiwa T. (2011): Phacoemulsification associated corneal damage evaluated by corneal volume. Am J Ophthalmol., 142:525528.

30. Taneri S, Oehler S, Lytle G and Dick B. (2014): Evaluation of epithelial integrity with various transepithelial corneal cross-linking protocols for treatment of keratoconus. J Ophthalmol., 2014:1-5.

31. Tawakol ME, Tag El-Din AE and Hassan AG. (2019): Corneal Hysteresis before and after Corneal Collagen Cross Linking for Keratoconus. The Egyptian Journal of Hospital Medicine, 77(5):5759-65.

32. Vinciguerra $P$, Albé $E$, Frueh BE, Trazza S and Epstein D. (2012): Twoyear corneal cross-linking results in patients younger than 18 years with documented progressive keratoconus. American Journal of Ophthalmology, 154(3):520-6.

33. Wollensak G, Spoerl E and Wilsch M. (2010): Keratocyte apoptosis after corneal collagen cross-linking using riboflavin/UVA treatment. Cornea, 23:43-49. 


\section{التغير ات في تقييم الخلايا المبطنة للقرنية بعد ربط الانسجة التعامدي للقرنية}

أحمد كمال عبد الفتاح فودة, محمد خضر محمد، عمر حسن سلامة قسم طب وجراحة العيون, كلية الطب، جامعة الأزهر، القاهرة، مصر

E-mail: ahmed.kamal.fouda@gmail.com

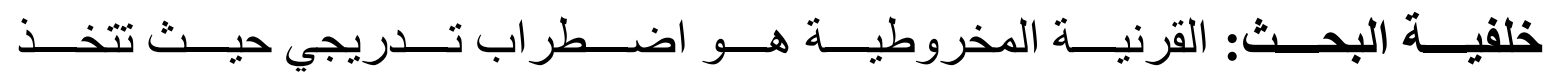

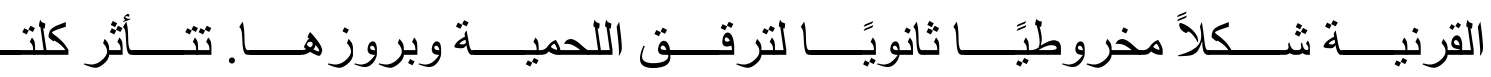

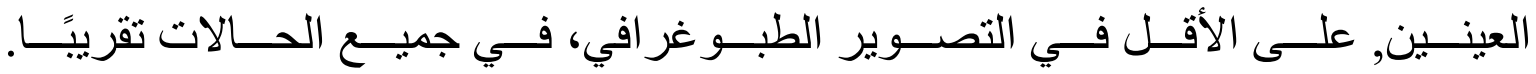

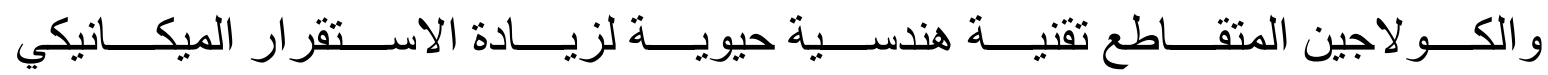
للقرنية في إضطر ابات القرنية الخارجية مثل القرنية المخروطية.

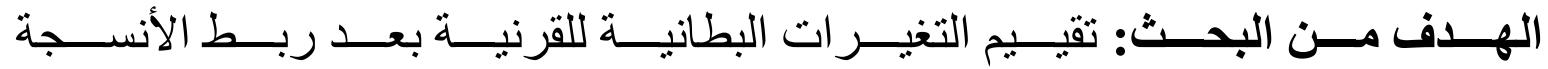

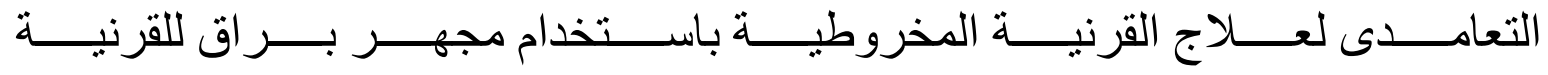
وقياسات حجم القرنية.

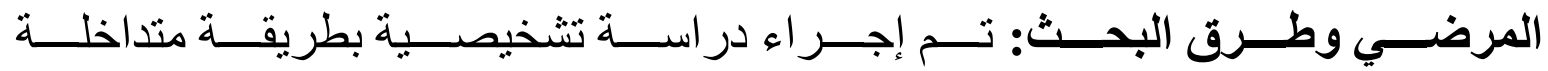

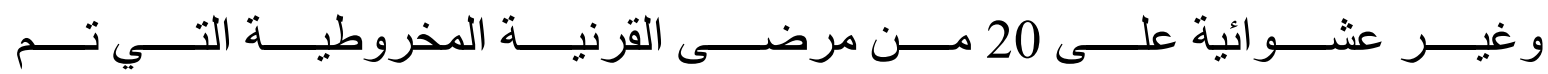

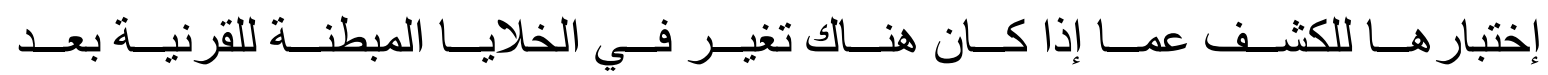

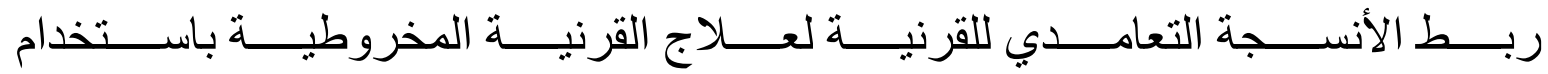

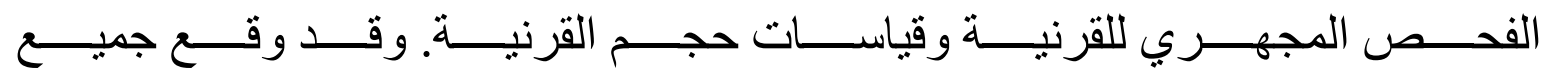

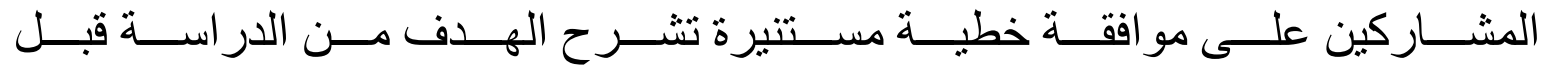

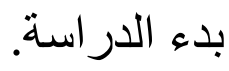

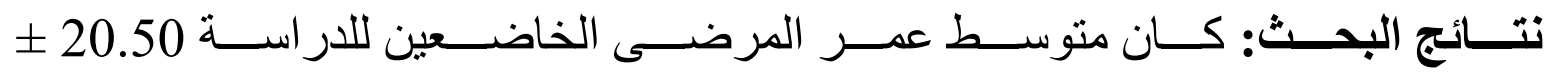

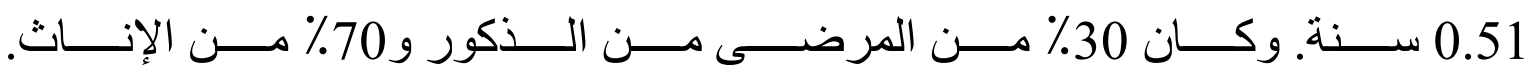

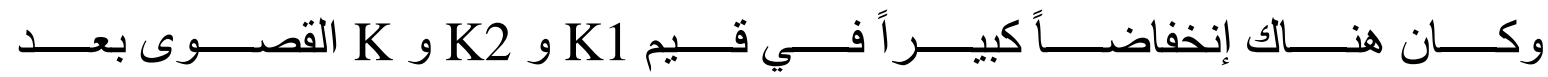




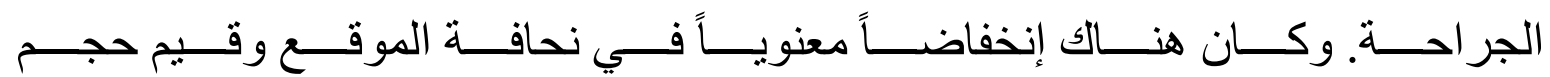

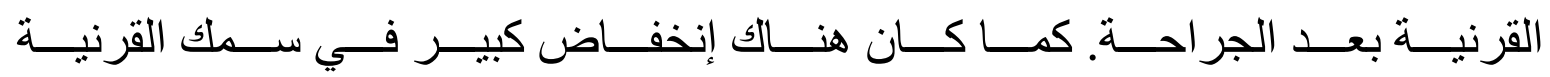

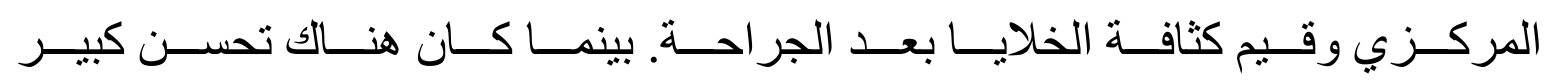

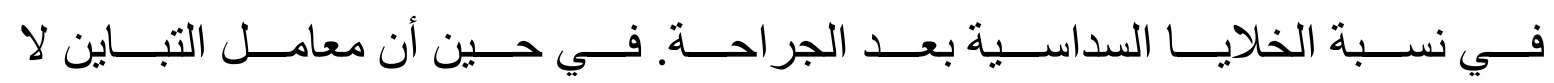

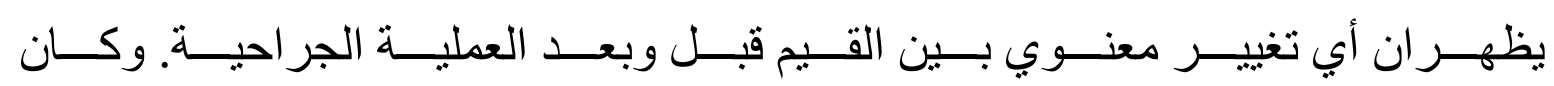

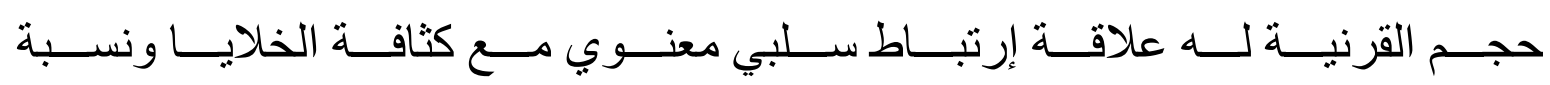
الخلايا السداسية, بينما لا يوجد إرتباط معنوي بمعامل التباين.

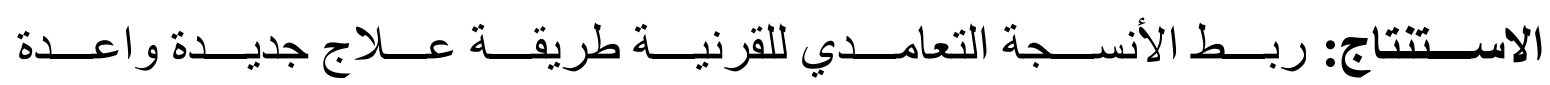

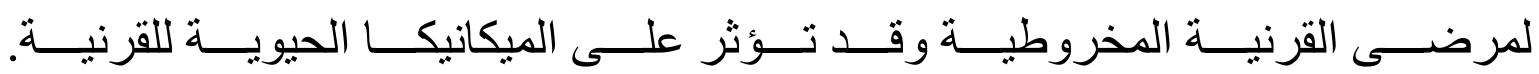

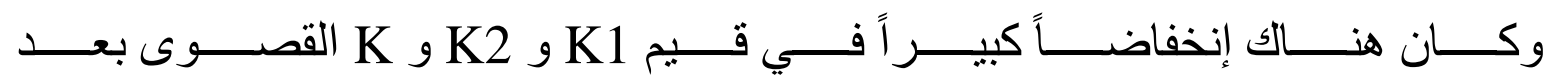

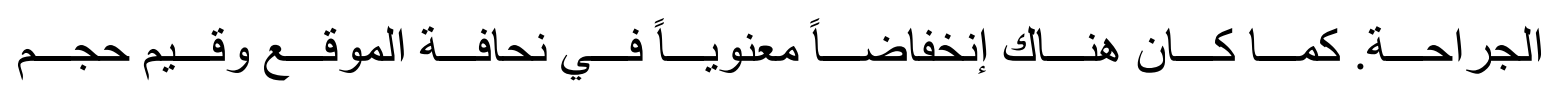
القرنية وسمك القرنية المركزي بعد الجراحة.

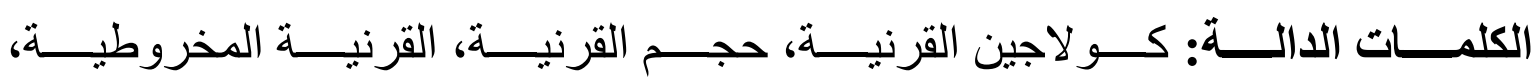

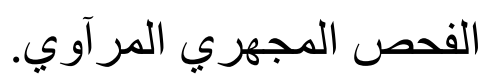

